\title{
Numerical method for prediction of duct break out sound power
}

Paul Williams, Ray Kirby, and James Hill

Citation: Proc. Mtgs. Acoust. 39, 022001 (2019); doi: 10.1121/2.0001205

View online: https://doi.org/10.1121/2.0001205

View Table of Contents: https://asa.scitation.org/toc/pma/39/1

Published by the Acoustical Society of America

\section{ARTICLES YOU MAY BE INTERESTED IN}

Dispersion based reduced-order model identification and boundary impedance control in a weakly coupled impedance tube

Proceedings of Meetings on Acoustics 39, 045005 (2019); https://doi.org/10.1121/2.0001215

Using Wikipedia to promote acoustics knowledge for the International Year of Sound 2020

Proceedings of Meetings on Acoustics 39, 025001 (2019); https://doi.org/10.1121/2.0001211

Numerical method for prediction of duct break out sound power

The Journal of the Acoustical Society of America 146, 3074 (2019); https://doi.org/10.1121/1.5137667

Study on impact of noise annoyance from highway traffic in Singapore City

Proceedings of Meetings on Acoustics 39, 015001 (2019); https://doi.org/10.1121/2.0001116

Finite element simulation and experiment in khaen acoustics

Proceedings of Meetings on Acoustics 39, 035003 (2019); https://doi.org/10.1121/2.0001178

Application of elastic parabolic equation solutions to calculation of acoustic reverberation in ice-covered underwater environments

Proceedings of Meetings on Acoustics 39, 022002 (2019); https://doi.org/10.1121/2.0001210

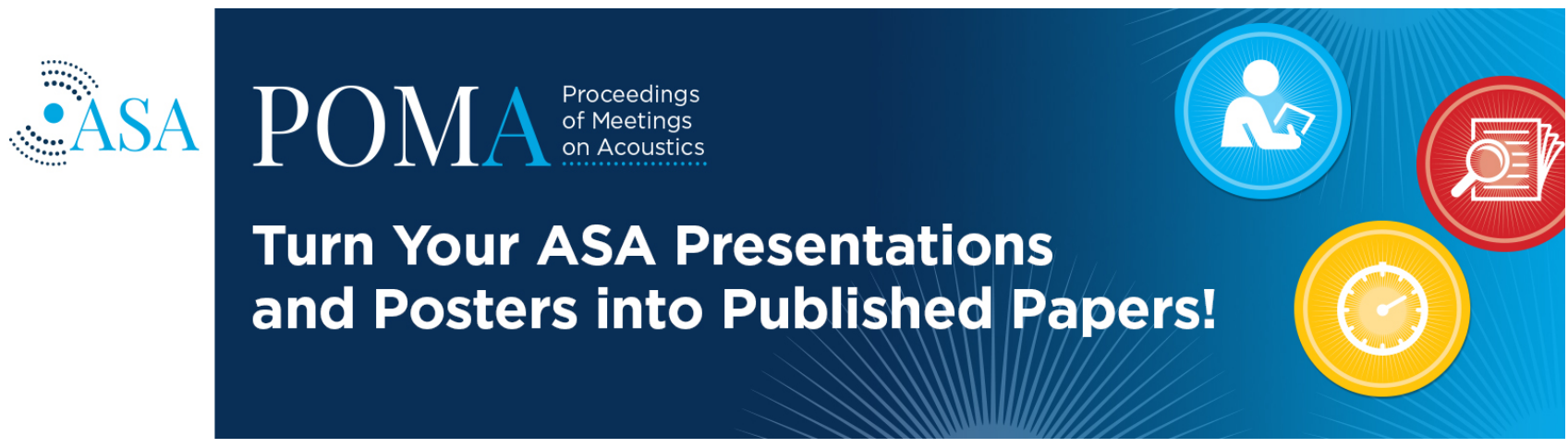




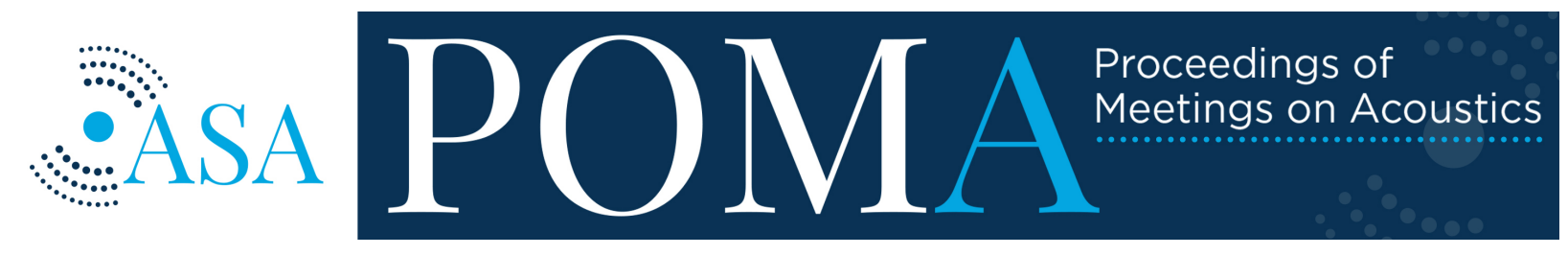

Volume 39

http://acousticalsociety.org/

\title{
178th Meeting of the Acoustical Society of America
}

San Diego, CA

2-6 December 2019

\section{Computational Acoustics: Paper 5aCA6}

\section{Numerical method for prediction of duct break out sound power}

\author{
Paul Williams \\ Faculty of Engineering and Information Technology, University of Technology Sydney, Ultimo, NSW, 2007, \\ AUSTRALIA; paul.williams@uts.edu.au
}

\author{
Ray Kirby \\ University of Technology Sydney, Sydney, NSW, AUSTRALIA; ray.kirby@uts.edu.au
}

\section{James Hill}

AAF Ltd, Cramlington, Northumberland, UNITED KINGDOM; james.hill@aafgb.com

The acoustic design of duct systems requires consideration of both the noise propagating within a duct and also of the noise transmitted out through the duct walls into the environment. Control of this breakout noise can form a significant part of a noise control solution, and so understanding this phenomenon can lead to reduced material use. The breakout noise is investigated here using coupled structural-acoustic finite element models. Propagation along a waveguide with constant cross section is represented using a modal expansion of the acoustic pressure in the fluid and displacement in the duct walls. The free-field external environment requires an outer boundary condition, and for this purpose, a perfectly matched layer is applied at some distance from the elastic walls. The finite length of the waveguide is then enforced by coupling the fields to separate infinite length inlet and outlet ducts by the mode matching method. Transmitted power from the finite length duct is investigated when a noise source is placed in the inlet.

Published by the Acoustical Society of America 


\section{INTRODUCTION}

Sound power breaking out of duct work can make up a significant amount of the noise pollution on industrial sites. Control of this noise is of importance during system design so that noise specifications are met. Prediction of this noise emitted from a duct therefore needs to be accurate.

Previous work has been carried out by Astley, Cummings and Sormaz ${ }^{1}$ and Cummings and Astley ${ }^{2}$ into various single skinned and lined duct geometries. In this work the structure was assumed to be a thin plate characterised by its surface displacement. It was shown that finite element methods were capable of accurately predicting the coupled acoustic-structural systems. Jade and Venkatesham ${ }^{3}$ studied the modal characteristics of a rectangular duct at low frequencies and also the radiated sound power. The above studies use a thin plate assumption for their models and this was shown to be appropriate with good agreement to experiment for the thin, single skinned ducts of around of $1 \mathrm{~mm}$ thick. In practice ducts and exhaust walls can be multi-layered with elastic elements on the order of $10 \mathrm{~mm}$ thick. Further research is therefore needed to explore the transmission loss of such designs.

In this paper the semi analytical finite element (SAFE) method ${ }^{4}$ will be demonstrated for single skinned, axisymmetric circular ducts. The SAFE method has been demonstrated in the past for the study of defects within waveguides ${ }^{5}$ and acoustic-structural interaction in buried pipes. ${ }^{4}$ This method is of interest to large exhaust predictions as it uses a 1-dimensional mesh along the radius and then propagates fields along the axis using an analytical expression. This has advantages over full 3-dimensional descriptions through the potential saving to memory and time, especially for long ducts and high frequencies. Here the SAFE method will be used to calculate propagation within steel ducts and to determine the transmission loss of finite length sections of infinitely long ducts. The ducts considered are circular and axisymmetric made of a single layer of steel which is either $1 \mathrm{~mm}$ or $10 \mathrm{~mm}$ thick. These ducts will be excited by a monopole to show the behaviour of the duct under a complex acoustic source.

\section{THEORY}

The transmission loss of an infinite duct is calculated here using the SAFE method which has been demonstrated previously ${ }^{4,10-14}$ and so is not detailed here. The waveguide under investigation is illustrated in Fig. 1. The cross-section consists of an internal fluid region, $\Omega_{1}$, an elastic region, $\Omega_{2}$, an external fluid region, $\Omega_{3}$, and a fluid PML region, $\Omega_{4}$. A monopole source is located at $r=0, z=0$ and the planes $\Gamma_{1}$ and $\Gamma_{2}$ at $z_{1}$ and $z_{2}$ respectively denote the location where the break out transmission loss of the duct is calculated.

The SAFE method describes the fluid regions using acoustic pressure, $p$, and the elastic regions using acoustic displacement in the radial, $u_{r}$, and axial, $u_{z}$ directions. To simplify the analysis only the axisymmetic modes are used, setting the polar order to zero. The degrees of freedom can be expressed as a sum of modes

$$
\begin{aligned}
p^{\prime}(r, z, t) & =\sum_{n=0}^{\infty} A_{n} p_{n}(r) \mathrm{e}^{i \omega t-i k_{r} \gamma_{n} z} \\
u_{r}^{\prime}(r, z, t) & =\sum_{n=0}^{\infty} A_{n} u_{r n}(r) \mathrm{e}^{i \omega t-i k_{r} \gamma_{n} z} \\
u_{z}^{\prime}(r, z, t) & =\sum_{n=0}^{\infty} A_{n} u_{z n}(r) \mathrm{e}^{i \omega t-i k_{r} \gamma_{n} z}
\end{aligned}
$$

where $\omega=2 \pi f, f$ is frequency, $k_{r}$ is a reference wavenumber, $\gamma_{n}$ is the normalised complex wavenumber, $n$ denotes the mode, $A_{n}$ is the amplitude of mode $n$. 

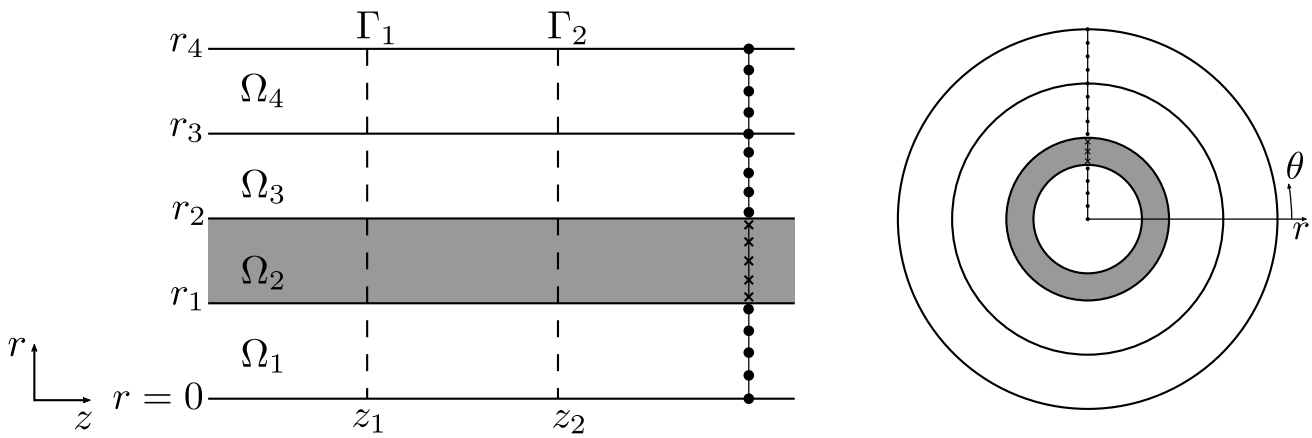

Figure 1: Diagram of the r-z plane of the axisymmetric waveguide and example finite element mesh. Nodes in the fluid are denoted by dots while nodes in the elastic are denoted by crosses.

To calculate the eigenvalues, $\gamma$, and eigenvectors, $p_{n}(r), u_{r n}(r)$ and $u_{z n}(r)$, the problem is formed into an eigenequation. This begins with the wave equations for the fluid domain and elastic region, given here as

$$
\begin{aligned}
& \frac{1}{c_{0}^{2}} \frac{\partial^{2} p^{\prime}}{\partial t^{2}}-\nabla^{2} p^{\prime}=0 \\
& \frac{\partial \sigma_{r r}^{\prime}}{\partial r}-i k_{r} \gamma \sigma_{r z}^{\prime}+\frac{\sigma_{r r}^{\prime}}{r}=-\rho_{1} \omega^{2} u_{r}^{\prime} \\
& \frac{\partial \sigma_{r z}^{\prime}}{\partial r}-i k_{r} \gamma \sigma_{z z}^{\prime}+\frac{\sigma_{r z}^{\prime}}{r}=-\rho_{1} \omega^{2} u_{z}^{\prime}
\end{aligned}
$$

where $\sigma_{r r}, \sigma_{r z}$, and $\sigma_{z z}$ are the elements of the Cauchy stress tensor, $\rho_{0}$ and $\rho_{1}$ is the density of air and steel respectively, and $c_{0}$ is the speed of sound in air. The wave equations are multiplied by a weighting function and integrated over the domain as in the usual finite element derivation and combined using suitable boundary conditions. The geometry is included through a 1-dimensional mesh along the radius using quadratic linear elements. The degrees of freedom are discretised as $p_{n}(r)=\mathbf{N}(r) \mathbf{p}_{n}$ where $\mathbf{N}(r)$ is a vector of shape functions expressed at each node and $\mathbf{p}_{n}$ is a vector at each node for mode $n$. Note that the elastic region is not approximated as a thin plate; the wave equation is used to express the displacement at each node across the thickness.

At the shared boundaries between fluid and elastic domains continuity of normal stress

$$
\begin{aligned}
\sigma_{r r} & =-p \\
\sigma_{z r} & =0
\end{aligned}
$$

and normal acoustic velocity

$$
\frac{\partial p}{\partial r}=\rho \omega^{2} u_{r}
$$

must be enforced. At the outer boundary of $\Omega_{3}$ the domain must be bounded considering an infinite space and so a perfectly matched layer (PML) is applied as shown by Duan and Kirby. ${ }^{4}$ Application of the finite element method to these coupled equations results in a set of quadratic simultaneous equations. The eigenproblem is arranged in the form $\mathbf{K d}=\gamma \mathbf{M d}$ where $\mathbf{d}=\left[\mathbf{p}, \mathbf{u}_{r}, \mathbf{u}_{z}, \gamma \mathbf{p}, \gamma \mathbf{u}_{r}, \gamma \mathbf{u}_{z}\right]^{T}$ which may be solved to find $\gamma, \mathbf{p}, \mathbf{u}_{r}$, and $\mathbf{u}_{z}$.

A monopole source is applied by setting the source amplitudes equal to

$$
\mathbf{A}=\frac{1}{\omega} \boldsymbol{\Lambda}^{-1} \mathbf{p}^{T} \mathbf{Q}
$$


where $\mathbf{Q}=\left[Q_{1}, Q_{2}, \cdots, Q_{N}\right]^{T}$ is a vector of nodal source amplitudes set to zero at all nodes except the monopole where it is equal to $Q_{0} / 2, \mathbf{A}=\left[A_{1}, A_{2}, \ldots, A_{N}\right]^{T}, \boldsymbol{\Lambda}$ is a matrix with elements $\Lambda_{n m}=$ $\int\left(p_{n} U_{z m}\right) r d r d \theta$, and $U_{z}$ is fluid displacement.

Using the source amplitudes and eigensolutions the sound power travelling along the waveguide at $\Gamma_{1}$ and radiated by the outer surface of the duct between $z_{1}$ and $z_{2}$ may be calculated. The sound power is calculated using the regular definitions for sound power in fluids and elastics ${ }^{7}$

$$
\begin{gathered}
W_{s}=\sum_{n}\left|A_{n}\right|^{2}\left(\frac{\pi k_{r}}{\omega \rho_{0}} \Re\left\{\gamma_{n}\right\} \int_{0}^{r_{1}}\left|p_{n}\right|^{2} r d r+\frac{\omega}{2} \Re\left\{i \int_{r_{1}}^{r_{2}}\left(\sigma_{r z n} u_{r n}^{*}+\sigma_{z z n} u_{z n}^{*}\right) r d r\right\}\right) \\
W_{r}=\frac{\pi r}{2 \omega \rho_{0} k_{r}} \sum_{n}\left|A_{n}\right|^{2} \Re\left\{\left(p_{n} \frac{\partial p_{n}^{*}}{\partial r}-p_{n}^{*} \frac{\partial p_{n}}{\partial r}\right) \frac{\mathrm{e}^{i k_{\mathrm{ref}}\left(\gamma_{n}^{*}-\gamma_{n}\right) z_{2}}-\mathrm{e}^{i k_{\mathrm{ref}}\left(\gamma_{n}^{*}-\gamma_{n}\right) z_{1}}}{\left(\gamma_{n}^{*}-\gamma_{n}\right)}\right\}
\end{gathered}
$$

where superscipt $*$ denotes a complex conjugate. The transmission loss, $L$, is defined as being the difference between the incident sound power, $W_{s}$, over the internal fluid and elastic region at $z_{1}$ and the sound power radiated, $W_{r}$, by the outer surface of the waveguide at $r_{2}$ between $z_{1}$ and $z_{2}$

$$
L=10 \log _{10}\left(\frac{W_{s}}{W_{r}}\right)
$$

\section{DUCT TRANSMISSION LOSS}

The transmission losses of single skinned steel ducts are investigated in this section. A monopole source is applied at $r=0$ and $z=0$ to an air filled steel pipe surrounded by air with the following radial dimensions; $r_{1}=500 \mathrm{~mm}, r_{2}=501 \mathrm{~mm}, r_{3}=2 \mathrm{~m}$, and $r_{4}=3 \mathrm{~m}$. The number of elements in each region is $n_{1}=60, n_{2}=20, n_{3}=200$, and $n_{4}=60$ for regions $1-4$ respectively. The steel has the properties $\rho_{1}=7850 \mathrm{kgm}^{-3}, c_{T}=3194 \mathrm{~ms}^{-1}$, and $c_{L}=5778 \mathrm{~ms}^{-1}$. The air has the properties $\rho_{0}=1.2 \mathrm{kgm}^{-3}$, and $c_{0}=344 \mathrm{~ms}^{-1}$. The resulting pressure and velocity this source generates will be shown. Following this the breakout of two ducts will be shown using transmission losses and the radiated power is then illustrated using the acoustic pressure.

At $f=1000 \mathrm{~Hz}$ the magnitude of the acoustic pressure, $\log _{10}|\Re(p)|$, and axial particle velocity, $\log _{10}\left|\Re\left(v_{z}\right)\right|$, on the source plane, $z=0$, is illustrated in Fig. 2. The pressure shows an expected maximum at the source location and demonstrates a non-planar mode pattern across the duct, which is expected as there are three propagating duct modes at this frequency. The effect of the duct wall at $r_{1}$ is clear with the pressure decreasing by over two orders of magnitude between the inner and outer wall of the duct. Pressure then reduces as it radiates away from the duct until $r_{3}$ at which point the magnitude rapidly decreases in the PML, providing a non-reflective boundary. It can be seen that the axial velocity reduces towards zero away from the monopole source $(r=0)$ as expected in order to enforce continuity of normal acoustic velocity across the source plane.

The transmission loss of the duct is predicted for a steel thickness of $1 \mathrm{~mm}, r_{2}=501 \mathrm{~mm}$, and $10 \mathrm{~mm}$, $r_{2}=510 \mathrm{~mm}$, with $z_{1}=1 \mathrm{~m}$ and $z_{2}=2 \mathrm{~m}$, see Fig. 3. As is expected for these ducts the transmission loss of the $10 \mathrm{~mm}$ duct wall is higher than that of the $1 \mathrm{~mm}$ duct wall with similar characteristics shared by both cases. Two regions can be observed in the transmission loss separated into the region below the ring frequency $(1630 \mathrm{~Hz})$, and the region above the ring frequency. Below the ring frequency the transmission loss decreases with frequency while above the ring frequency the transmission loss increases with frequency. Minima can be seen across the spectrum at frequencies corresponding to the duct modes cutting-on within the internal fluid; the first of these being at $419 \mathrm{~Hz}$. At these frequencies the transmission loss is lower, however the minima are narrow and so have only a small effect on transmission loss. 

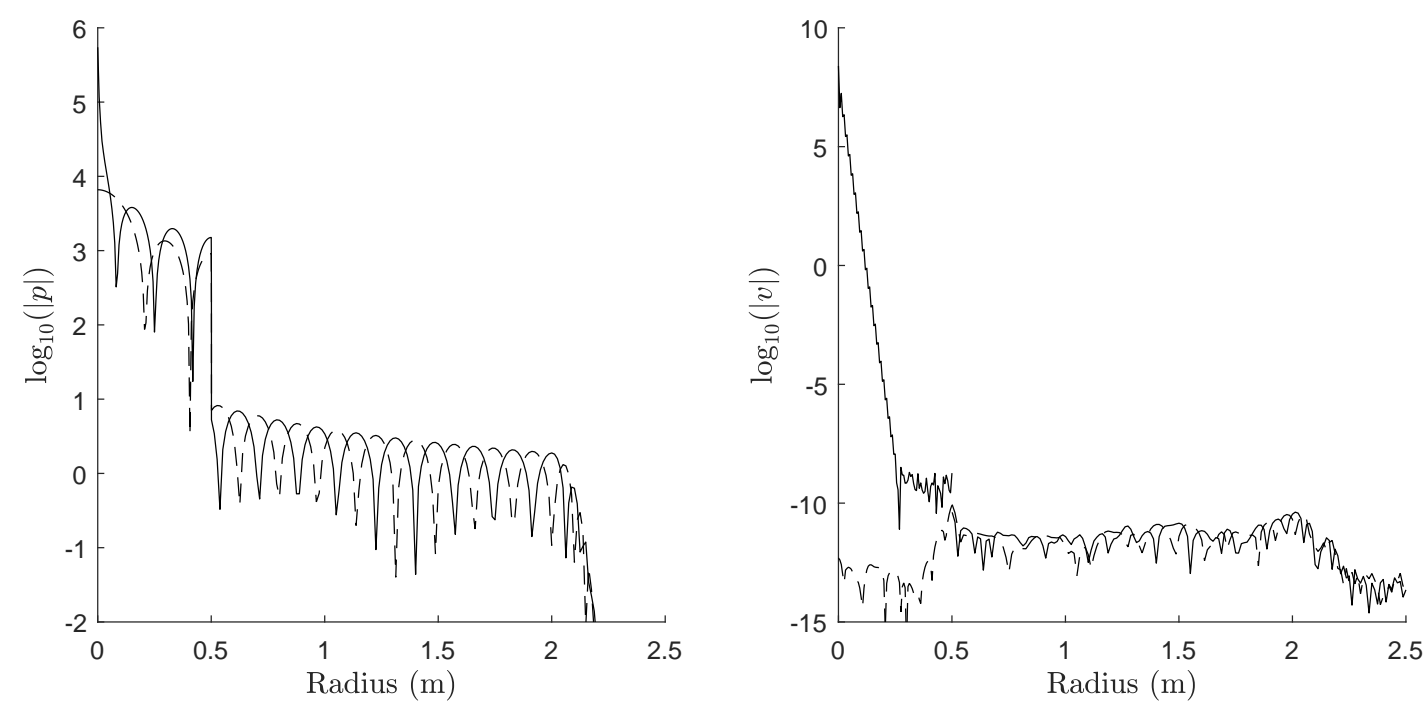

Figure 2: Acoustic pressure, left, and axial velocity, right, on the source plane. _—, real; - - -, imaginary.

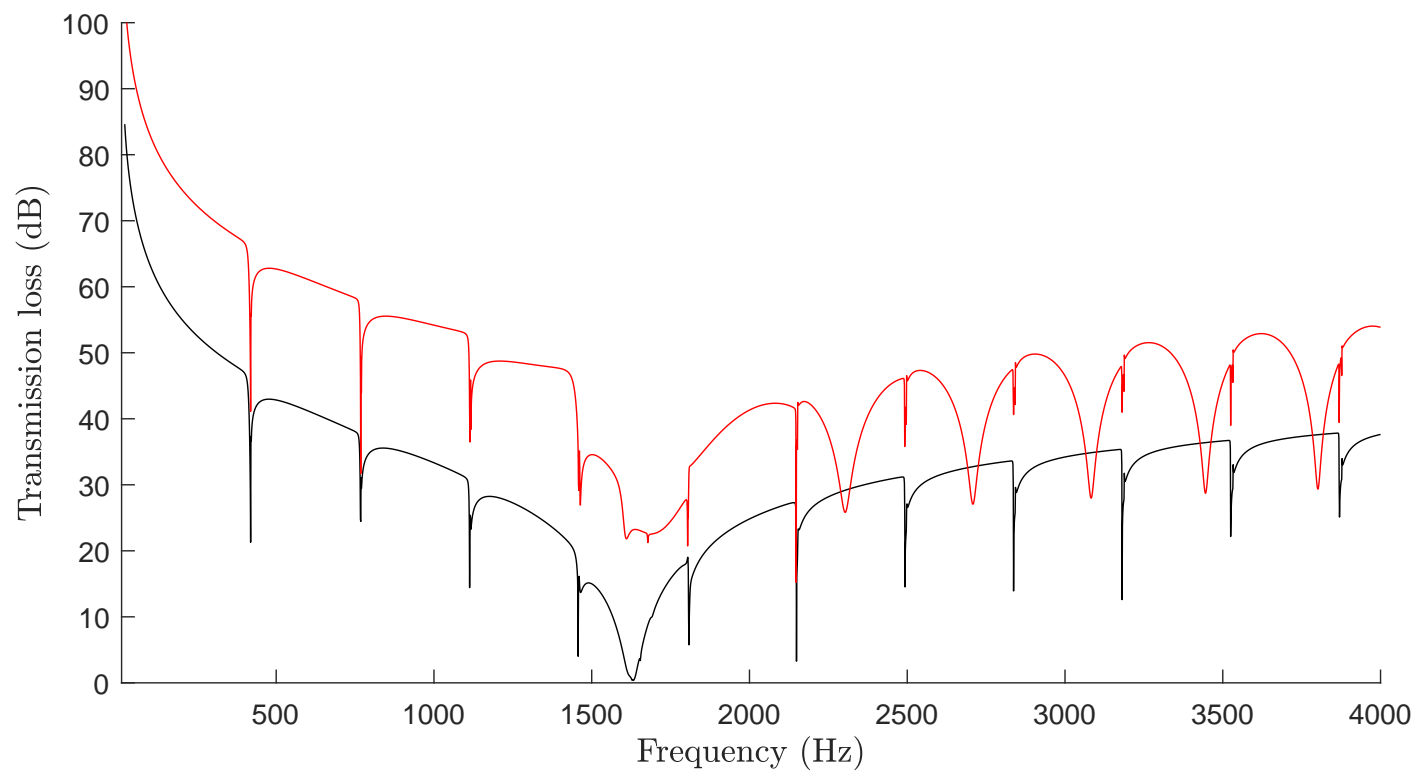

Figure 3: Transmission loss of the duct with a steel thickness of $1 \mathrm{~mm}$ ( - ) and 10mm (- $\longrightarrow$. 


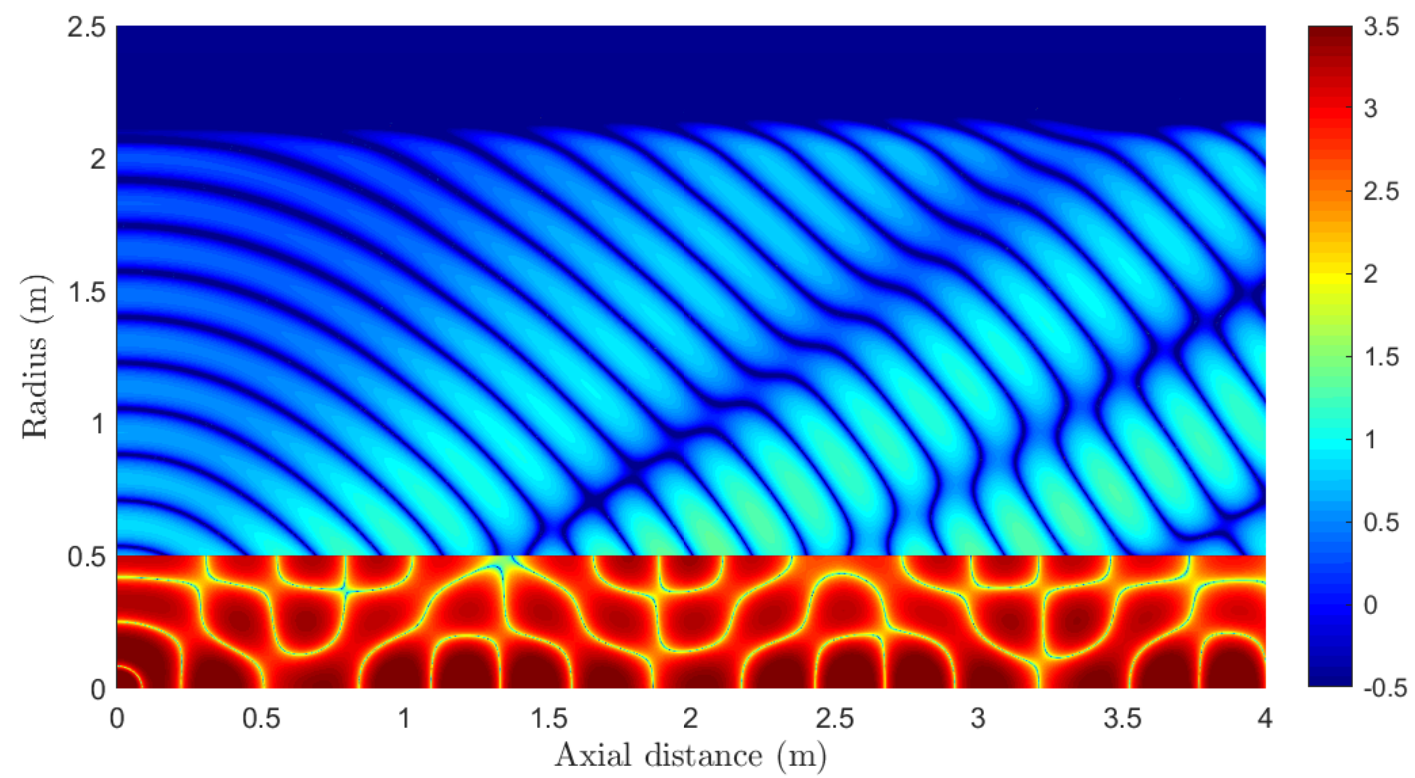

Figure 4: Magnitude of the acoustic pressure and $\sigma_{r r}$ in the radial-axial plane at $1000 \mathrm{~Hz}$ with a duct thickness of $1 \mathrm{~mm}$.

The magnitude of the real part of the pressure field, $\log _{10}|\Re\{p\}|$, at $1000 \mathrm{~Hz}$ is illustrated in Fig. 4. At this frequency there are three propagating modes inside the duct but it is below the ring frequency. The monopole therefore excites a multi-modal pressure field within the duct and there is a low level of radiation from the duct surface. It can be seen that this radiated pressure creates a non planar field in the external fluid region where the maximum radiated pressure is not found along the source plane, but instead appears at a distance from the source.

At the ring frequency the transmission loss of the $1 \mathrm{~mm}$ duct approaches zero, see Fig. 3 . Here the importance of including the energy in both the elastic and internal fluid region is found. If the energy within the elastic region is not included in Eq. (11) then the transmission loss of the duct wall becomes negative for the $1 \mathrm{~mm}$ thick duct. This implies that the sound power does not immediately pass through the duct wall when radiated but instead travels along the wall prior to break out. The effect of this high transmission is illustrated in Fig. 5 for $\log _{10}|\Re\{p\}|$. Pressure levels are similar inside and outside the duct and the lack of insulation given by the pipe results in an increasingly planar field within the duct. This increasingly planar field is a result of the loss of energy from the pipe and it is interesting to compare this to the internal pressure at $1000 \mathrm{~Hz}$, see Figure 4, where the internal sound pressure is less planar despite being at a lower frequency.

Above the ring frequency a set of minima are found for the $10 \mathrm{~mm}$ case at $2300 \mathrm{~Hz}, 2700 \mathrm{~Hz}, 3080 \mathrm{~Hz}$, $3450 \mathrm{~Hz}$, and $3800 \mathrm{~Hz}$. These minima are relatively broadband and cause the transmission loss to drop below the transmission loss of the $1 \mathrm{~mm}$ duct. At these minima the duct wall is effectively transmitting sound pressure at a particular angle such as is expected for coincidence frequencies, see Fig. 6. Beranek ${ }^{8}$ gives the critical frequency, at which the structure's bending wave and the acoustic wavelength are equal, as $f_{c}=c_{0}^{2} /\left(1.8 c_{L}\left(r_{2}-r_{1}\right)\right)$ so that $f_{c}=1140 \mathrm{~Hz}$ for the $10 \mathrm{~mm}$ duct wall. Using this equation shows that $f_{c}\left(r_{2}-r_{1}=1 \mathrm{~mm}\right)=11400 \mathrm{~Hz}$ is above our region of interest but $f_{c}\left(r_{2}-r_{1}=10 \mathrm{~mm}\right)=1140 \mathrm{~Hz}$ is within the frequency range allowing for these features to occur.

The transmission loss of the $10 \mathrm{~mm}$ thick duct is calculated at $2 \mathrm{~m}$ lengths, $z_{2}-z_{1}=2 \mathrm{~m}$, at various distances from the source, $z_{1}$. This distance between source and transmission section causes changes in the minima associated with the duct's cut-on frequencies but otherwise has no effect on transmission loss. These 


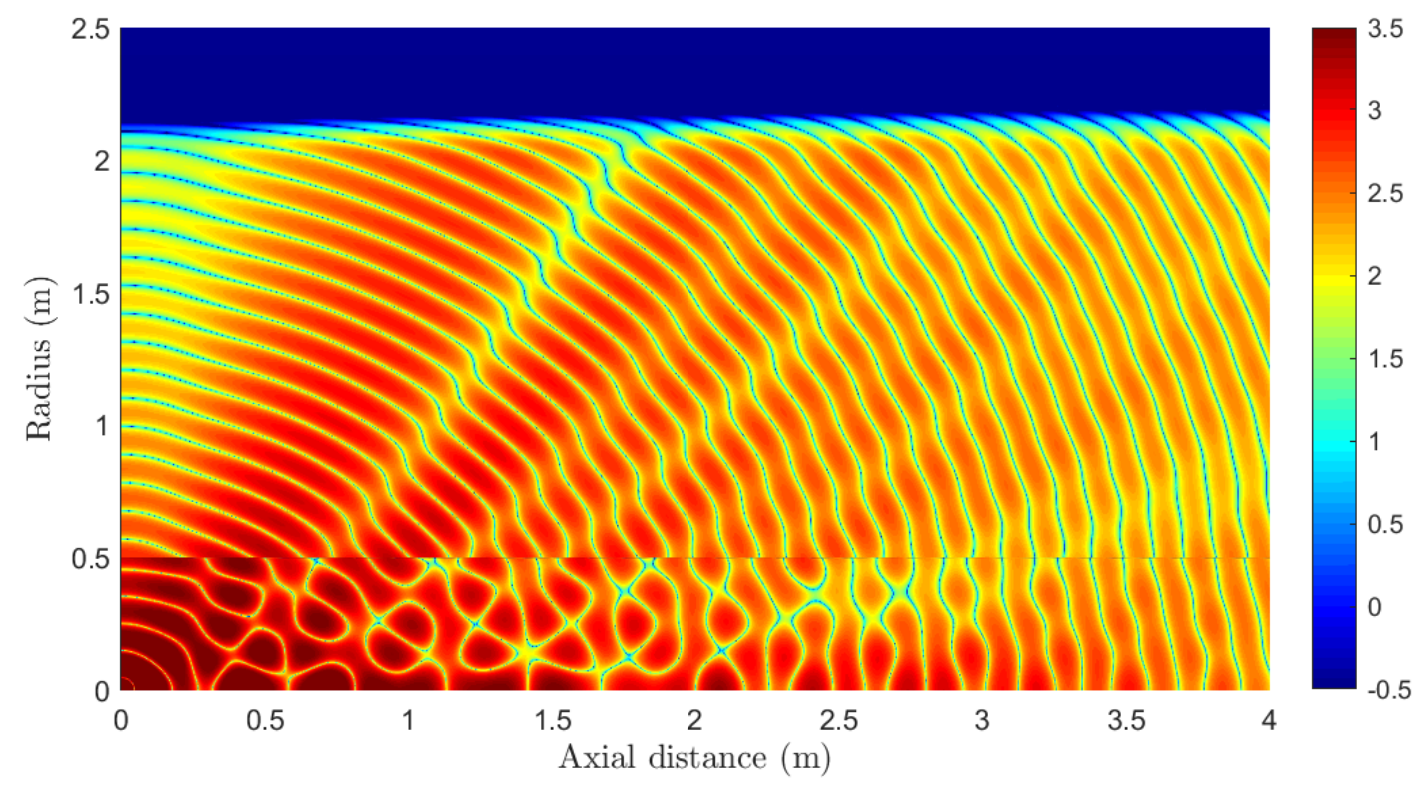

Figure 5: Magnitude of the acoustic pressure and $\sigma_{r r}$ in the radial-axial plane at $1630 \mathrm{~Hz}$ for a $1 \mathrm{~mm}$ duct thickness.

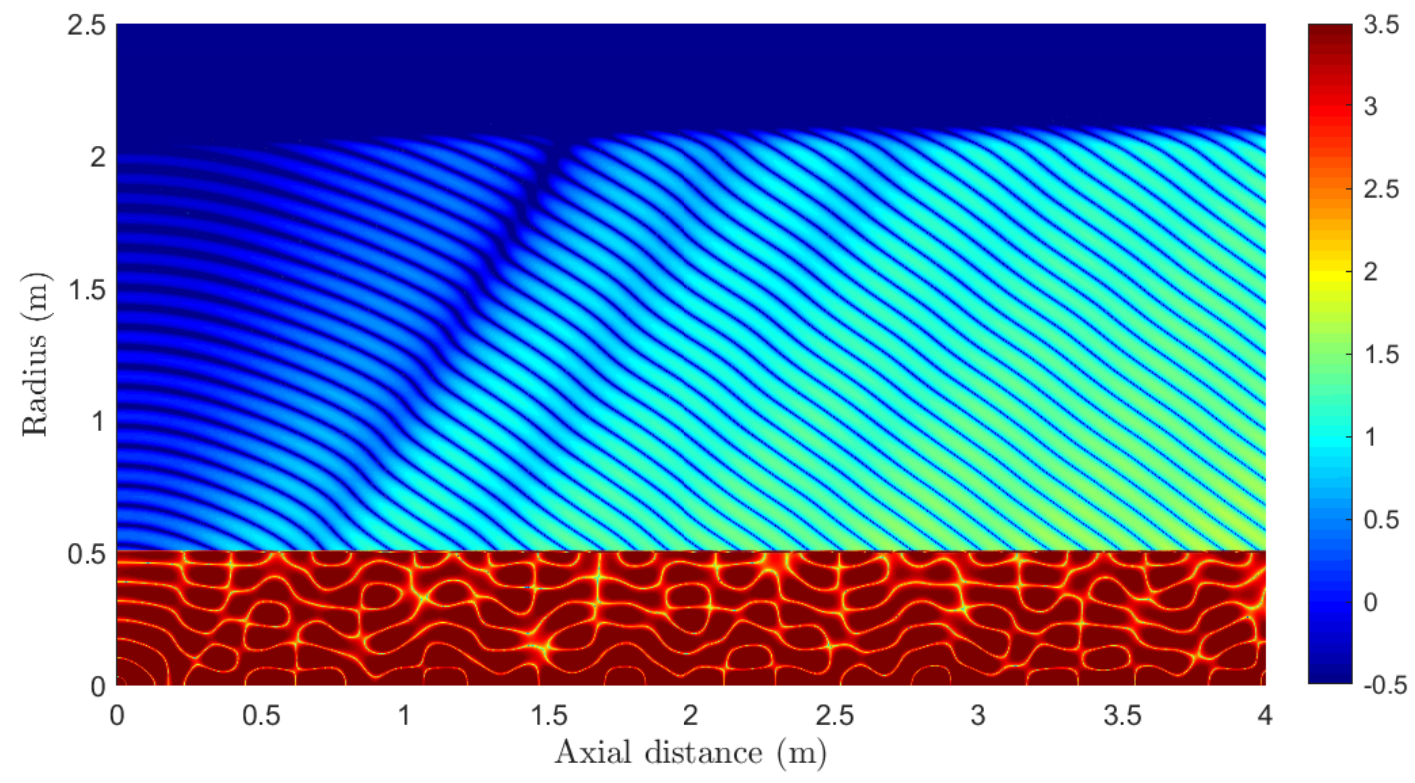

Figure 6: Magnitude of the acoustic pressure and $\sigma_{r r}$ in the radial-axial plane at $2300 \mathrm{~Hz}$ for a $10 \mathrm{~mm}$ duct thickness. 
minima become narrower with lower reductions as $z_{1}$ increases. This change occurs on the low frequency side of the cut-on frequency where the mode has not fully cut-on inside the duct and is still imaginary. Longer distances between the source and transmission section therefore cause the energy in the nearly cuton mode to be more attenuated, lowering the amount of power available to be radiated. This has the effect of slightly increasing transmission loss in octave bands containing a minima at longer distances from the source, however the effect is generally small and decreases with distance. Calculating the transmission loss at small values of $z_{1}$ will therefore give a more conservative calculation of transmission loss as the energy of evanescent duct modes is included in the radiated sound power.

The above work has been concerned with infinite length ducts with uniform cross-section. However in duct systems there are regular changes caused by the varying sound reduction needs, flanges and other elements. A method is therefore needed to connect these different cross-sections together at their interfaces. Once method which may be used to do this is the mode matching method. This has been demonstrated for coupling uniform wavegudies to finite element domains when predicting silencer performance. ${ }^{9}$ A similar method may be applied for waveguides involving elastic and fluid regions.

\section{CONCLUSION}

In this paper the transmission loss of an axisymmetric duct with uniform cross-section is investigated. Wave propagation through the system is predicted using a semi-analytical finite element method modelling the stress throughout the elastic structure without assuming it to be a thin plate. Predictions are generated for two ducts having wall thicknesses of $1 \mathrm{~mm}$ and $10 \mathrm{~mm}$ and their break out transmission loss is calculated along a finite length. It is demonstrated that increasing wall thickness generally increases transmission loss as expected, although minima at characteristic frequencies can cause thicker duct walls to exhibit higher losses. The pressure field is illustrated at various frequencies demonstrating the change to the radiated field. The method demonstrated in this paper is a first step towards the modelling of multilayered duct structures and finite length sections.

\section{ACKNOWLEDGMENTS}

This research was funded by the Australian Government through the Australian Research Council (project number LP170100881).

\section{REFERENCES}

${ }^{1}$ R.J. Astley, A. Cummings and N. Sormaz, "A finite element scheme for acoustic propagation in flexiblewalled ducts with bulk-reacting liners, and comparison with experiment", Journal of Sound and Vibration 150, 119-138 (1991).

2 A. Cummings and R.J. Astley, "The effects of flanking transmission on sound attenuation in lined ducts", Journal of Sound and Vibration 179, 617-646 (1995).

${ }^{3}$ N. Jade and B. Venkatesham, "Experimental study of breakout noise characteristics of flexible rectangular duct", Mechanical Systems and Signal Processing 108, 156-172 (2018).

${ }^{4}$ W. Duan and R. Kirby, "Guided wave propagation in buried and immersed fluid-filled pipes: Application of the semi analytic finite element method", Computers and Structures 212, 236-247 (2019).

${ }^{5}$ W. Duan, R. Kirby, and P. Mudge, "On the elastic scattering of elastic waves from a non-axisymmetric defect in a coated pipe", Ultrasonics 65, 228-241 (2016). 
${ }^{6}$ C.L. Scandrett and C.L. Frenzen, "Bi-orhtogonality relationships involving porous materials", J. Acoust. Soc. Am. 98, 1199-1203 (1995).

${ }^{7}$ L. Gavric, U. Carlsson, and L. Feng, "Measurement of structural intensity using a normal mode approach", Journal of Sound and Vibration 206, 87-101 (1997).

${ }^{8}$ L.L. Beranek, "Noise reduction”, 2nd reprint ed, Peninsula Pub, Los Altos, Calif (1991).

${ }^{9}$ P. Williams, R. Kirby, J. Hill, M. Åbom and C. Malecki, "Reducing low frequency tonal noise in large ducts using a hybrid reactive-dissipative silencer", Applied Acoustics 131, 61-69 (2018).

${ }^{10}$ W. Duan, R. Kirby, P. Mudge and T.-H. Gan, "A one dimensional numerical approach for computing the eigenmodes of elastic waves in buried pipelines", Journal of Sound and Vibration 384, 177 - 193 (2016).

${ }^{11}$ A. Marzani, E. Viola, I. Bartoli, F. L. di Scalea and P. Rizzo, "A semi-analytical finite element formulation for modeling stress wave propagation in axisymmetric damped waveguides", Journal of Sound and Vibration 318, 488 - 505 (2008).

${ }^{12}$ P. W. Loveday, "Semi-analytical finite element analysis of elastic waveguides subjected to axial loads", Ultrasonics 49, 298 - 300 (2009).

${ }^{13}$ L. Gavrić, "Computation of propagative waves in free rail using a finite element technique", Journal of Sound and Vibration 185, 531 - 543 (1995).

${ }^{14}$ D. Inoue and T. Hayashi, "Transient analysis of leaky Lamb waves with a semi-analytical finite element method”, Ultrasonics 62, 80 - 88 (2015). 